\title{
Increased amygdala reactivity following early life stress: a potential resilience enhancer role
}

\author{
Tetsuya Yamamoto, 2,10, Shigeru Toki ${ }^{3}$, Greg J. Siegle ${ }^{1,4}$, Masahiro Takamura ${ }^{3}$, Yoshiyuki Takaishi ${ }^{3}$, \\ Shinpei Yoshimura ${ }^{5}$, Go Okada ${ }^{3}$, Tomoya Matsumoto ${ }^{3}$, Takashi Nakao ${ }^{6}$, Hiroyuki Muranaka ${ }^{7}$, Yumiko Kaseda ${ }^{8}$, \\ Tsuneji Murakami ${ }^{9}$, Yasumasa Okamoto ${ }^{3^{*}}$ and Shigeto Yamawaki ${ }^{3}$
}

\begin{abstract}
Background: Amygdala hyper-reactivity is sometimes assumed to be a vulnerability factor that predates depression; however, in healthy people, who experience early life stress but do not become depressed, it may represent a resilience mechanism. We aimed to test these hypothesis examining whether increased amygdala activity in association with a history of early life stress (ELS) was negatively or positively associated with depressive symptoms and impact of negative life event stress in never-depressed adults.

Methods: Twenty-four healthy participants completed an individually tailored negative mood induction task during functional magnetic resonance imaging (fMRI) assessment along with evaluation of ELS.

Results: Mood change and amygdala reactivity were increased in never-depressed participants who reported ELS compared to participants who reported no ELS. Yet, increased amygdala reactivity lowered effects of ELS on depressive symptoms and negative life events stress. Amygdala reactivity also had positive functional connectivity with the bilateral DLPFC, motor cortex and striatum in people with ELS during sad memory recall.

Conclusions: Increased amygdala activity in those with ELS was associated with decreased symptoms and increased neural features, consistent with emotion regulation, suggesting that preservation of robust amygdala reactions may reflect a stress buffering or resilience enhancing factor against depression and negative stressful events.
\end{abstract}

Keywords: Early life stress, Amygdala reactivity, fMRI, Resilience, Depression

\section{Background}

Increased reactivity to emotional information is characteristic of depression, and has been linked with increased and sustained reactivity in the amygdala [1-4]. Hyperreactivity is often associated with vulnerability to depression as it occurs in populations that tend to become depressed such as children with anxiety or depressed parents [5] as well as those at risk for depressive relapse [6], those with early life stress (ELS) [7], cognitively vulnerable individuals [8], and individuals with inhibited

\footnotetext{
*Correspondence: oy@hiroshima-u.ac.jp

${ }^{3}$ Department of Psychiatry and Neurosciences, Institute of Biomedical and Health Sciences, Hiroshima University, 1-2-3 Kasumi, Minami-ku, 734-8551 Hiroshima, Japan

Full list of author information is available at the end of the article
}

temperament [9]. That said, not all individuals with high levels of vulnerability become depressed. Rather, we will consider whether amygdala hyper-reactivity, as a consequence of early stress, may contribute to resilience against developing depression in otherwise vulnerable individuals. This is important as intervening on hyperreactivity prior to the onset of depression would be either indicated or contra-indicated based on its causal role.

A specific vulnerability factor for depression, history of ELS, has been linked to both amygdala hyper-reactivity [10] and hypo-reactivity [11]. Effects of stress on the amygdala [12] are hypothesized to underlie alterations in cognition, mood, and behavior [13-15]. These changes have been further hypothesized to shape individual differences in vulnerability for mood and anxiety disorder, 
such as emotional reactivity [16-18]. That said, there is scant evidence for this complete causal pathway.

Rather, a great deal of data shows that early life stressors are associated with increased amygdala reactivity in the absence of psychiatric diagnoses [15]. This could represent vulnerability for future depression, or could suggest that neural adaptations to stress are protective.

In fact, decreased amygdala activity may be a vulnerability factor. For example, patients with borderline personality disorder are vulnerable to depression and frequently display decreased amygdala activity during emotional challenges [19]. The capacity to react to emotional information is hypothesized to be protective with blunted reactivity being more clearly associated with pathology [20] including depression [21].

Here we suggest that in vulnerable individuals, robust amygdala reactivity may be protective compared to more blunted amygdala reactivity. Adaptive responses to stressors in childhood can have a "stress inoculating" effect, and lead to resilience to future stressors [22]. To clarify the role of amygdala reactivity in resilience, we examined, using functional magnetic resonance imaging (fMRI), whether increased amygdala activity downmodulated depressive symptoms and the impact of life events in individuals with a history of ELS but no history of depression. Since our goal was to examine the extent to which ELS might confer vulnerability for future depression via increasing depressive severity within a subclinical range, we recruited healthy people who did not have severe depressive symptoms. Subclinical depressive severity was interpreted as an index of vulnerability [23]. Also, to examine potential mechanisms for preserved reactivity we further explored functional connectivity with the amygdala in this sample.

\section{Methods}

\section{Participants}

Thirty healthy volunteers, who did not have any physical and mental problems, were recruited through adverts in local newspapers and public notices. All participants were interviewed by a psychiatrist or psychologist to assess for a health condition and a lifetime history of DSM-IV psychiatric disorders using the MINI International Neuropsychiatric Interview [24, 25]. Exclusion criteria were as follows: being left-handed or ambidextrous [26], history of seizures, head trauma, use of psychotropic medications, magnetic resonance imaging (MRI) contraindications or technical alterations, unsuccessful induction of negative mood and participants who exceed the cutoff score for depressive symptoms (Beck Depression Inventory II >29) [27, 28]. All participants scored in the normal range on a cognitive screen $[29,30]$, with a verbal IQ-equivalent $>80$. We excluded three participants in whom the negative mood induction failed to produce an increase in negative mood or decrease in positive mood, two who exceeded the cutoff score for depressive symptoms $[27,28]$, and one who showed excessive head movement ( $>3.0 \mathrm{~mm}$ over the functional MRI run). Thus, 24 participants (6 male) were included in the analyses. One participant was missing fMRI data from one rest block. As such, their data was included in analyses of block-related averages but not in time-series analyses for which complete data were required.

The study was approved by the Research Ethics Committee of Hiroshima University. After complete description of the study to the participants, written informed consent was obtained. Participants received 5,000 Japanese yen ( US\$42) to compensate them for their time.

\section{Self-report Measures}

We assessed early life stress (ELS) using the Japanese version of the Child Abuse and Trauma Scale (CATS) $[31,32]$. The CATS is a 38-item retrospective self-report questionnaire that measures subjective perception of four ELS subtypes (negative home environment/neglect, sexual abuse, punishment, and emotional abuse). Participants rated how frequently they experienced particular adverse events experience during their childhood and adolescence using a five-point scale $(0=$ never, $4=$ always $)$. Scores for each factor are calculated based on the mean value of the individual items for each subscale, and range between 0 and 4 . Higher mean values represent more severe ELS. The CATS has favorable psychometric properties, including adequate test-retest reliability, internal consistency, and concurrent validity [31, 33]. As shown in Table 1, participants reported similar CATS scores to those in a previous study that used healthy undergraduate students [31]. Scores generally fell within what is described by the measure's authors as the mild to moderate range.

Table 1 Demographic and Subjective Data

\begin{tabular}{lrll}
\hline Measure & Mean & (SD) & [Range] \\
\hline Age (years) & 40.70 & $(11.05)$ & {$[24-60]$} \\
Verbal Intelligence & 110.36 & $(6.62)$ & {$[97-120]$} \\
PHQ-9 & 3.67 & $(3.78)$ & {$[0-14]$} \\
CATS Total Score & 0.84 & $(0.60)$ & {$[0.2-2.5]$} \\
$\quad$ Negative Home Environment/Neglect & 0.85 & $(0.64)$ & {$[0-2.8]$} \\
$\quad$ Sexual Abuse & 0.10 & $(0.18)$ & {$[0-0.7]$} \\
$\quad$ Punishment & 1.51 & $(0.74)$ & {$[0-2.7]$} \\
$\quad$ Emotional Abuse & 0.89 & $(0.85)$ & {$[0-2.9]$} \\
LES Impact of Negative Life Event & 2.58 & $(4.86)$ & {$[0-23]$} \\
Difficulty to recall sad memory & 3.21 & $(2.77)$ & {$[0-9]$} \\
Vividness of recalled sad memory & 6.08 & $(2.87)$ & {$[1-10]$} \\
\hline
\end{tabular}

CATS Childhood Abuse and Trauma Scale, LES Life Experiences Survey, PHQ-9 Patient Health Questionnaire-9 
Participants' mood states during the scanning session were assessed using a computer based Visual Analogue Scale (VAS) (Fig. 1). Participants rated their moods on three unipolar VAS measuring happiness, sadness, and anxiety dimensions by moving a cursor with a four button response pad. The VAS dimensions were projected onto a screen in the MR scanner. The scales ranged from 0 to 100 , with 0 indicating "not at all" and 100 indicating "extremely". In a debriefing session after scanning, we asked participants to rate the extent to which they were successfully engaged in the mood induction task (which required recall of personal experiences) on two 11-point scales (0-10). Participants rated "difficulty to recall memory," and "vividness of the recalled memories."

We assessed the impact of recent negative life events using the Japanese version of the Life Event Stress Scale (LES) [34, 35]. For the LES, participants were asked to indicate which of 57 events had occurred during the previous 12 months, and to rate the impact of each event using a seven-point scale, ranging from extremely negative $(-3)$ to extremely positive $(+3)$. The total scores for impact of negative life events were used.

Current symptoms of depression were assessed using the Japanese version of the Patient Health Questionnaire9 (PHQ-9) [36, 37]. Verbal intelligence was estimated using the Japanese National Adult Reading Test [29, 30].

\section{Mood induction paradigm}

To develop an individualized, negative mood inducing fMRI task, we used a combination of re-experiencing personal emotional episodes and listening to music associated with sad mood. We modified a mood induction paradigm developed by Ramel et al. [6] and Segal et al. [38], which was used in an individualized version of a block design paradigm.
For the memory recall procedure, participants were asked to write four detailed autobiographical scripts about two very sad personal experience (sad memory) and two specific but unemotional days in their lives (neutral memory). On a scale from 1 (neutral) to 9 (extremely sad), they were encouraged to describe sad episodes that they rated 5 or higher. For the neutral memory, participants were also asked to write in detail about a specific but unemotional day in their lives. The scripts were sent to participants about one week prior to the session day.

In a pre-scanning session, participants were asked to listen to the first minute of four music pieces on a PC. Using a computer-based VAS, they rated (a) the degree to which the music created a sad impression and (b) the degree to which the music was able to bring about sadness. The VAS ranged from 0 to 100 , with 0 indicating "not at all" and 100 indicating "extremely". The music consisted of a standard selection battery [39], which has been used in multiple mood induction studies [6, 40, 41]. These included: Russia under the Mongolian Yoke composed by Sergei Prokefiev, played at half speed; Adagio for Strings composed by Samuel Barber; Peer Gynt - The Death of Ase, composed by Edvard Grieg; and Adagio in $G$ Minor composed by Tomaso Albinoni. The sad memory recall procedure was accompanied by the two sad music selections that were rated most highly on each VAS for each individual. The neutral recall condition was accompanied by the musical pieces Venus, the Bringer of Peace and Neptune, the Mystic by Gustav Holst.

To ensure that an appropriate mood was induced in participants, we used an altered block design paradigm (Fig. 1) in which a standardized $30 \mathrm{~s}$ block from the same emotional set of stimuli commenced only after the participant indicated (via a button press) that they were

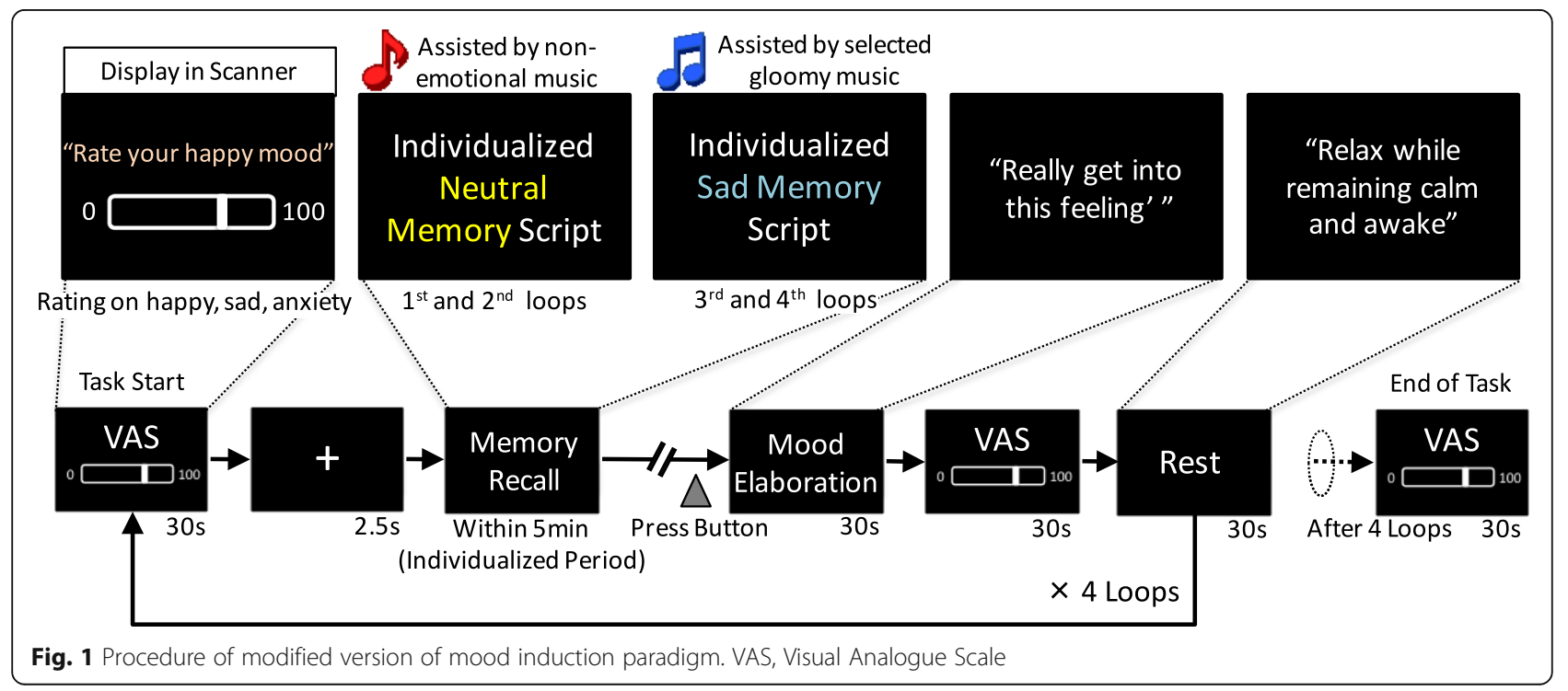


clearly experiencing a neutral or sad mood. While in the MR scanner, participants listened to the selected musical piece (presented via headphones) while reading and attempting to re-experience the sadness of the event depicted in the autobiographical script, which was projected onto a screen. Each musical piece and autobiographical script was presented for up to $5 \mathrm{~min}$ (an individualized recall period) before each of the $30 \mathrm{~s}$ blocks. The duration of presentation depended on the time it took for the participant to achieve the appropriate mood state. Music was not played during the $30 \mathrm{~s}$ block periods.

All participants were exposed to eight $30 \mathrm{~s}$ alternating blocks of recall (sad or neutral) followed by resting periods, in addition to the individualized recall period. Participants' mood state was assessed using a VAS during the scanning session. Participants were first asked to recall an emotionally neutral memory and then a sad memory. On the basis of a previous study [42], this design was adopted to avoid contamination of the neutral stimuli by the sad stimuli.

\section{fMRI image acquisition}

Images were acquired using a 3.0 T MRI scanner (SIGNA HDxt; GE; single-shot, echo planar imaging (EPI) with whole-brain coverage, 32 axial slices per $2500 \mathrm{~ms}$ TR, TE $=30 \mathrm{~ms}$, flip angle $=90^{\circ}$, matrix size $=$ $64 \times 64, \mathrm{FOV}=240 \mathrm{~mm}$, slice thickness $=4 \mathrm{~mm}$, interslice gap $=0 \mathrm{~mm}$ ). A high resolution $\mathrm{T} 1$-weighted image provided anatomical localization (Ir-P FSPGR; TE = $1.9 \mathrm{~ms}, \mathrm{TR}=6.9 \mathrm{~ms}$, flip angle $=20^{\circ}$, matrix size $=$ $256 \times 256, \quad \mathrm{FOV}=25.6 \mathrm{~mm}$, slice thickness $=1 \mathrm{~mm}$, inter slice gap $=0 \mathrm{~mm}, 180$ slices).

\section{fMRI data preprocessing}

Preprocessing and analysis of fMRI data were conducted using the statistical parametric mapping software package, SPM 8 (Wellcome Department of Cognitive Neurology, London, UK). The first 4 volumes of the fMRI run were discarded to ensure a steady-state MR signal. Time-series were slice-time corrected, volume registered to the mean image, and coregistered with T1-weighted structural images. T1 images were bias-corrected and segmented using the International Consortium for Brain Mapping (ICBM) tissue probability maps for gray matter, white matter, and cerebrospinal fluid. Time-series data were spatially normalized to the ICBM152 template, smoothed with an $8 \mathrm{~mm}$ FWHM Gaussian kernel, and high-pass filtered at $0.008 \mathrm{~Hz}$.

\section{Behavioral data analyses}

To test whether participants showed both greater increases in sadness and greater reductions in happiness after sad memory recall than after neutral memory recall, we used a two-way repeated measures analysis of variance (ANOVA). We used two within-subject factors (mood: happy, sad, and anxious; time: baseline /VAS1, post- $1^{\text {st }}$ neutral memory recall $[\mathrm{NR}] / \mathrm{VAS} 2$, post- $1^{\text {st }}$ rest/VAS3, post- $2^{\text {nd }}$ NR/VAS4, post- $2^{\text {nd }}$ rest/VAS5, post$1^{\text {st }}$ sad memory recall [SR] /VAS6, post- $3^{\text {rd }}$ rest/VAS7, post- $2^{\text {nd }}$ SR/VAS8, and post- $4^{\text {th }}$ rest/VAS9). To control for Type I errors across the analyses, we used the Bonferroni procedure. Significance level was set at $p<0.05$. One participant was excluded from the analysis due to missing data following a scanner problem.

To examine the relationships between mood change, ELS, depression, the impact of recent negative life events, and the degree of task engagement, we conducted a Pearson's correlation analysis. Mood change (the effect of mood induction on mood) was operationalized by comparing mood after sad memory recall with mood after neutral memory recall, i.e., mood change $=([$ VAS6 + VAS8 $]-[$ VAS2 + VAS4 $]) / 2$. Significance level was set at $p<0.05$ (two-tailed).

All behavioral analyses were performed using SPSS v. 22.0 (SPSS Japan Inc., Tokyo, Japan).

\section{fMRI data analyses}

To visualize the amygdala region activated during sad memory recall, preprocessed time series data for each participant were analyzed using multiple regression. We measured amygdala activity during the sad/neutral mood elaboration period (30 s; Fig. 1). The model included a regressor for the contrast term 'sad mood recall vs. neutral mood recall'. Thus, for each voxel, amygdala reactivity $=B O L D_{\text {Sad }}$ Recall $-B O L D_{\text {Neutral Recall }}$. Given our focus on amygdala reactivity, amygdala activation was examined using small volume correction. We used a statistical threshold of $p<0.05$, family wise error (FWE) corrected, for the bilateral amygdala with an extent threshold of 10 contiguous voxels. The amygdala was defined according to Tzourio-Mazoyer et al. [43], and one bilateral amygdala mask was created using the WFU PickAtlas [44]. In a second step, the mean contrast values for the significant cluster from the initial analysis were extracted using the MarsBaR region of interest (ROI) toolbox (version 0.43) [45], and further analyzed using SPSS 22 (SPSS Japan Inc., Tokyo, Japan). Subsequent analyses were performed using mean contrast values, except for two analyses of the time-series of amygdala activity and the generalized psychophysiological interaction (gPPI) analysis.

We conducted Pearson's correlation analyses using each of the four CATS subscale scores and amygdala activity, to examine the association of history of ELS and amygdala responsiveness. We also evaluated a hierarchical multiple regression model predicting amygdala reactivity using the CATS scores, age, PHQ-9 scores, LES negative 
life events scores, and task engagement (difficulty recalling a memory and the vividness of the recalled memory). Significance for correlations was set at $p<0.05$ (two-tailed).

We also investigated the differences in the time-series of amygdala activity between people with a past history of ELS and people with no history. Activity in the significant clusters within the amygdala, derived from the mood induction task, were extracted and averaged using the 12 scans (30 s) preceding each VAS rating during the mood elaboration period. Activation in the amygdala was expressed as a percentage difference from a prestimulus baseline (VAS1). We performed a three-way repeated measures ANOVA with one between-subject factor (group: ELS and non-ELS) and two within-subject factors (mood; time). One non-ELS participant was excluded due to missing data following a scanner problem.

Finally, to examine the moderated effect of amygdala reactivity on depressive symptoms and the impact of negative life events in people with a history of ELS, we performed moderated multiple regression involving ELS as a predictor, amygdala reactivity as a moderator, and depression and impact of negative life events as dependent variables. To capture the way in which activity in other brain regions modulates amygdala reactivity, we used the gPPI toolbox [46]. The seed regions were significant clusters of activity in the bilateral amygdala that were identified in the preceding analysis for mood induction. As a task regressor, we used the contrast term 'sad mood recall - neutral mood recall'. The obtained individual gPPI images were used to perform a random effect analysis using a whole-brain two sampled t-test. The threshold of the gPPI analysis was set at $p<0.005$, uncorrected. Cluster k extent, determined by 1000 Monte Carlo simulations at the whole-brain level, was implemented in AlphaSim [47] for 290 voxels (for the left amygdala seed) and 253 voxels (for the right amygdala seed), and at cluster levels of $p<0.05$, corrected.

Each statistical threshold of fMRI data analysis was set on the basis of previous studies [10,11, 48].

\section{Results \\ Mood manipulation check}

Mood $\times$ time repeated measures ANOVA revealed a significant $\operatorname{mood} \times$ time interaction, Greenhouse-Geisser $F$ $(3.79,83.35)=24.73, p<.001, \eta_{\mathrm{p}}^{2}=.53$. Paired samples t-tests of change over time within conditions showed that after sad memory recall (VAS6 and VAS8), participants demonstrated significantly increased sadness ratings, and significantly decreased happiness ratings in comparison with baseline (VAS1) and before sad memory recall (VAS2, VAS3, VAS4, and VAS5), while anxiety ratings remained constant throughout the experiment (Fig. 2). Most of the participants were able to recall vividly both neutral memory and sad memory (Table 1 ). While some people reported the difficulty of recalling memory, we confirmed that their moods were successfully manipulated.

\section{Relationship between early life stress, mood change and amygdala reactivity Mood change}

There was a non-significant positive correlation between sad mood change and CATS sexual abuse scores, $r=.38$, $p=.071$, and a significant negative correlation between happy mood change and CATS sexual abuse, $r=-.51$, $p=.014$. There were no associations of mood change and other self-report measures, all $r s<-.20, p s>.368$.

\section{Amygdala reactivity}

There were significant bilateral amygdala regions for the sad vs neutral mood contrast, left amygdala, $x=-28$,

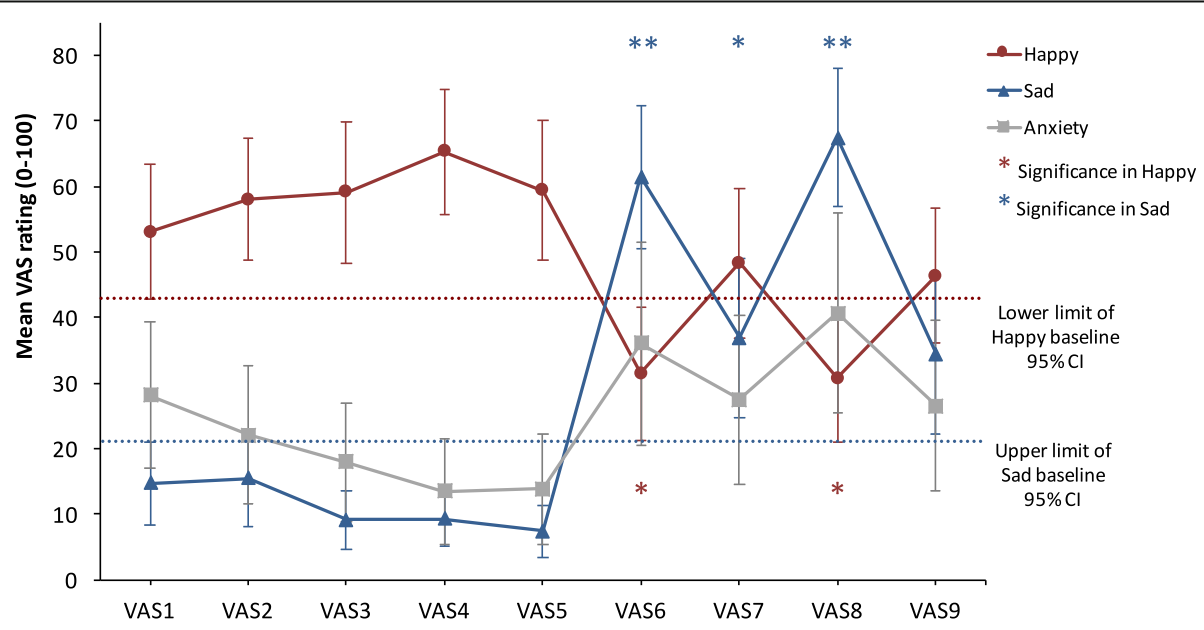

Fig. 2 Mood induction effects on mood. Error bars represent the 95\% confidence interval (CI). *,**, Significant difference from VAS1 (baseline) score $\left({ }^{*} p<0.05{ }^{* *} p<0.001\right.$, two-tailed); VAS1, baseline; VAS2, post- $1^{\text {st }}$ neutral memory recall (NR); VAS3, post- $1^{\text {st }}$ rest; VAS4, post- ${ }^{\text {nd }}$ NR; VAS5, post- $2^{\text {nd }}$ rest; $V A S 6$, post- $1^{\text {st }}$ sad memory recall (SR); VAS7, post- $3^{\text {rd }}$ rest; VAS8, post- $2^{\text {nd }}$ SR; $V A S 9$, post- $4^{\text {th }}$ rest 
$\mathrm{y}=-4, \quad \mathrm{z}=-22, \quad Z=3.65, p \quad$ FWE-corrected $=.001, \quad \mathrm{k}=34$, and right amygdala, $\mathrm{x}=28, \mathrm{y}=-4, \mathrm{z}=-20 . Z=3.92, p$ FWEcorrected $<.001, \mathrm{k}=68$.

CATS sexual abuse scores were significantly correlated with right amygdala activity, $r=.48, p=.018$. There was a non-significant correlation between CATS sexual abuse and left amygdala activity, $r=.38, p=.064$. The other CATS subscales did not significantly correlate with amygdala activity, all $r \mathrm{~s}<-.27, p \mathrm{~s}>.195$.

Hierarchical multiple regression analysis showed that sexual abuse scores significantly predicted right amygdala reactivity above and beyond other features, $\Delta F$ $(1,18)=9.81, \Delta R^{2}=.29, p=.006, \beta=.61$, and for the left amygdala reactivity, $\Delta F(1,18)=5.65, \Delta R^{2}=.22, p=.029$, $\beta=.53$. No other CATS subscale had significant effects, all $\Delta F \mathrm{~s}<1.82, p \mathrm{~s}>.195$.

\section{Influences of early life stress on time-series of mood change and amygdala activity}

Based on these results, additional analyses further addressed whether increased amygdala reactivity was likely to be protective or a vulnerability factor within the child sexual abuse group. We divided the participants into two groups: those who had reported child sexual abuse $(n=7$, 0 male; ELS group) and those without reported child sexual abuse ( $n=17,17$ male; non-ELS group). There were moderately strong but non-significant differences between these groups including age, $t(22)=1.02, p=.32, d=.46$,
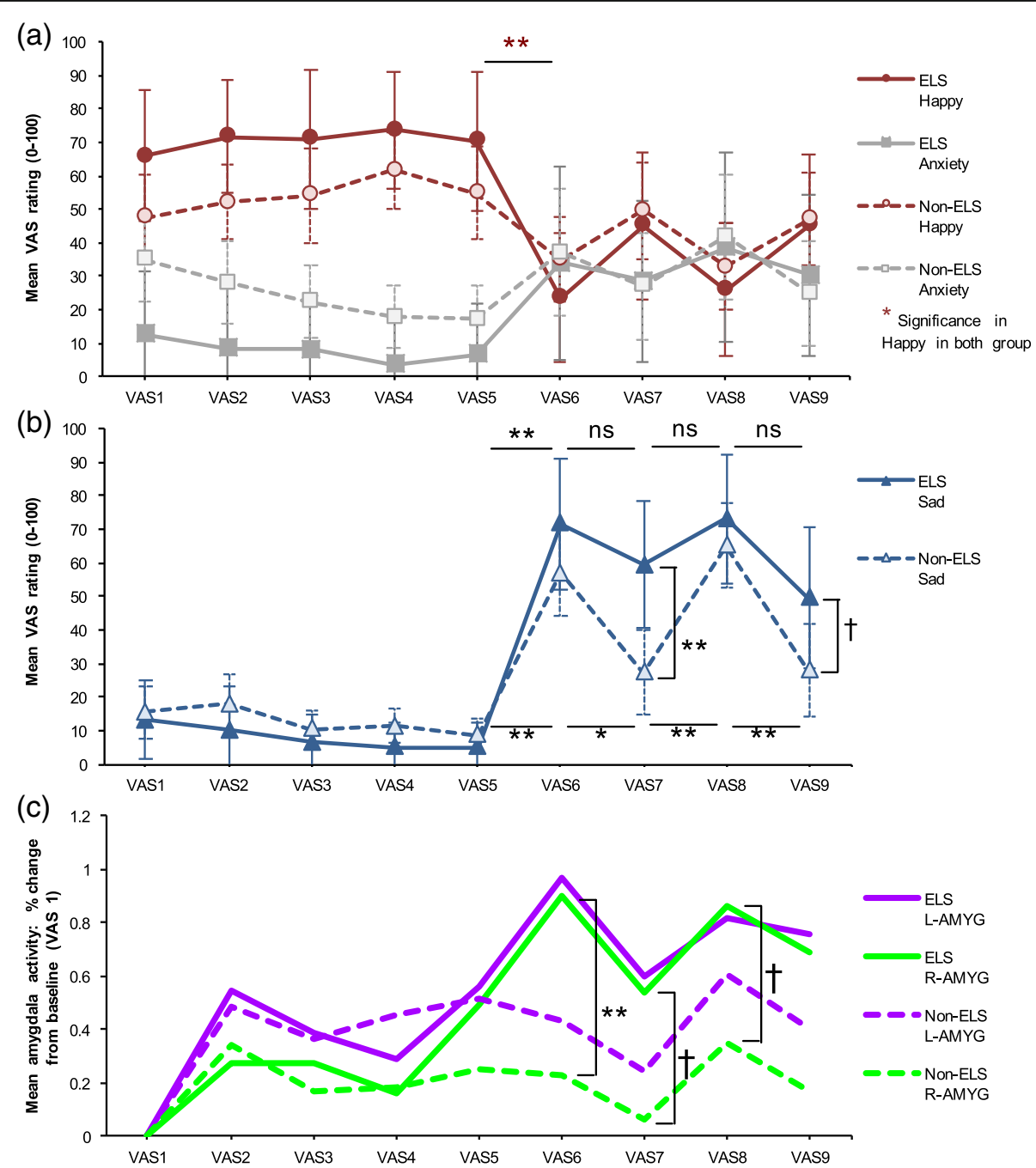

Fig. 3 Relationship among experience of early life stress, mood change, and amygdala activity. a Mood induction effects on happiness and anxiety in ESL group and non-ESL group. b Mood induction effects on sadness in ESL group and non-ESL group. $\mathbf{c}$ Time courses for right and left amygdala activity in significant cluster. Error bars represent the $95 \%$ confidence interval. ${ }^{*},{ }^{*}{ }^{*}$, Significant difference for a priori time points of interest $\left(t p<0.10,{ }^{*} p<0.05,{ }^{* *} p<0.01\right.$, two-tailed). VAS1, baseline; VAS2, post- $1^{\text {st }}$ neutral memory recall (NR); VAS3, post- ${ }^{\text {st }}$ rest; VAS4, post- $2^{\text {nd }}$ NR; VAS5, post-2 ${ }^{\text {nd }}$ rest; $V A S 6$, post- $1^{\text {st }}$ sad memory recall (SR); VAS7, post-3 ${ }^{\text {rd }}$ rest; VAS8, post-2 ${ }^{\text {nd }}$ SR; VAS9, post- $4^{\text {th }}$ rest; ESL, early life stress; L-AMYG, left amygdala; R-AMYG, right amygdala 
sex, $\chi^{2}(1)=3.29, p=.13, V=.36$, xdepressive symptoms, $t(7.78)=1.10, p=.30, d=.62$, negative life events, $t$ $(6.45)=1.32, p=.23, d=.87$, vividness of recalled memory, $t(22)=1.88, p=$. 073, $d=.84$, and difficulty of memory recall, $t(22)=1.59, p=.127, d=.71$.

\section{Time-series of mood}

Group (ELS and non-ELS) $\times$ Mood $\times$ Time repeated measures ANOVA revealed a significant three-way interaction, Greenhouse-Geisser $F(4.40,92.33)=3.47$, $p=.009, \eta_{\mathrm{p}}^{2}=.14$. As with Fig. 2, both groups demonstrated significantly increased sadness ratings, and significantly decreased happiness ratings after sad memory recall (VAS6 and VAS8; Fig. 3a, b). There were not any significant group differences for the happiness and anxiety at each time point, and these groups reported consistent changes across the experiment (Fig. 3a). However, ELS participants did not show significant decreases in sadness at the rest period after sad memory recall (VAS7 and VAS9) in comparison with the prior time point (VAS6 and VAS8), while non-ELS participants showed significantly decreased sadness at the same time point (Fig. 3b). Furthermore, ELS participants rated their sadness as higher than did non-ELS participants at the rest period after sad memory recall (VAS7 and VAS9).

\section{Time-series of amygdala activity}

Group (ELS and non-ELS) $\times$ Time repeated measures ANOVA showed significant main effects for time for both amygdala, left amygdala, Greenhouse-Geisser $F$ $(4.58,96.01)=3.18, p=.013, \eta_{\mathrm{p}}^{2}=.13$, and right amygdala, $F(8,168)=3.63, p<.001, \eta_{\mathrm{p}}^{2}=.15$, and a non-significant interaction for the right amygdala, $F(8,168)=1.95$, $p=.055, \eta_{\mathrm{p}}^{2}=.09$. As with the time course of sadness, for associations of amygdala over time, ELS participants had similar left and right amygdala activity,
VAS3-VAS9, $r \mathrm{~s}>.70, p \mathrm{~s}<.08$. Right amygdala activation in ELS participants was significantly or almost significantly higher after sad memory recall (VAS6 and VAS8) and during the rest period (VAS7) than in non-ELS participants (Fig. 3c). Non-ELS participants had higher activity on the left, slightly diminishing the significance of the group difference after sad memory recall during the rest period, VAS6-VAS9, $p \mathrm{~s}>.177, d \mathrm{~s}<.60$.

\section{Moderation Effects of Amygdala Reactivity on the Relationship between ELS and Symptom severity Depression}

There was a significant ELS interaction with the left amygdala, $\Delta F(1,20)=5.43, \Delta R^{2}=.20, p=.030, \beta=-.60$, and right amygdala, $\Delta F(1,20)=6.71, \Delta R^{2}=.23, p=.018$, $\beta=-.72$. Results remained significant when age and behavioral differences including the degree of difficulty in recalling memories and vividness of memory recall were covaried out. As shown in Fig. 4a, left and right high amygdala activity were associated with low predicted depression scores only for the highest ELS individuals $(+1 \mathrm{SD})$.

\section{Impact of negative stress events}

There was a significant ELS interaction with the left amygdala, $\Delta F(1,20)=12.03, \Delta R^{2}=.32, p=.002, \beta=-.75$, and right amygdala, $\Delta F(1,20)=4.77, \Delta R^{2}=.17, p=.041$, $\beta=-.61$. As shown in Fig. $4 \mathrm{~b}$, left and right high amygdala activity predicted low impact of negative life events only for the highest (+1SD) ELS scorers.

\section{Functional Connectivity of Amygdala with Other Brain Areas}

As summarized in Table 2 and Fig. 5, left amygdala activity in ELS people was mainly accompanied by increased functional interactions with bilateral DLPFC, bilateral motor cortex, and bilateral striatum. Associations of the right amygdala with these areas were of similar magnitude
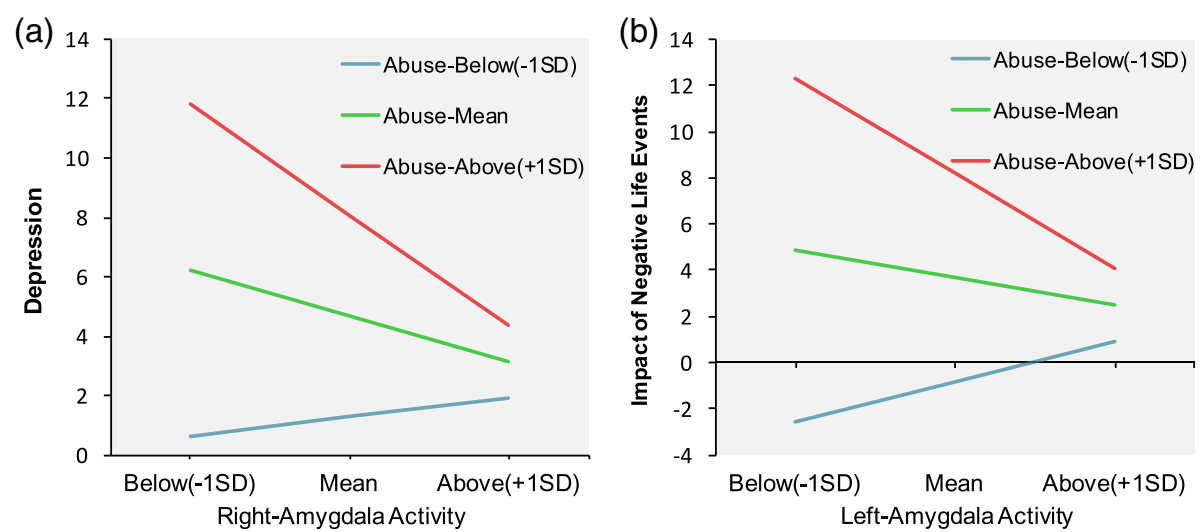

Fig. 4 Moderation effects of amygdala reactivity. a Slope of the relation between right amygdala activity and depression as a function of ELS. b Slope of the relation between left amygdala activity and impact of negative life events as a function of ELS. 
Table 2 Left amygdala functional connectivity in early life stress group during sad memory recall

\begin{tabular}{|c|c|c|c|c|c|c|c|}
\hline Region & Location of centroid voxel & Brodmann areas & $x$ & $y$ & z & size & Z \\
\hline L prefrontal cortex & $L$ inferior frontal gyrus & $46,45,10$ & -50 & 32 & 24 & 427 & 4.47 \\
\hline L motor cortex & L postcentral gyrus & $6,4,40,9,3$ & -52 & -8 & 36 & 551 & 4.45 \\
\hline R striatum & R putamen & - & 26 & 16 & 0 & 427 & 4.45 \\
\hline R mortor cortex & $\mathrm{R}$ postcentral gyrus & $6,2,3,4,40,43,1,41,13,9,22$ & 66 & -16 & 28 & 1086 & 4.45 \\
\hline R prefrontal cortex & $\mathrm{R}$ middle frontal gyrus & $9,10,46$ & 24 & 42 & 32 & 428 & 4.22 \\
\hline R striatum & L putamen & - & -22 & 4 & 6 & 295 & 4.12 \\
\hline
\end{tabular}

$L$ left, $R$ right

Note: Threshold was set at uncorrected $p<0.005$ for the volume of the whole brain, minimum extent 290 voxels, and at cluster levels of corrected $p<.05$

but were not significant after correction for multiple comparisons.

\section{Discussion}

We examined the effects of ELS and amygdala reactivity to mood challenge on symptoms and the impact of negative life events in healthy participants screened for current and past psychiatric conditions. Negative mood induction successfully elicited amygdala activation in addition to increased sadness and decreased happiness. Consistent with the prevailing literature [49], ELS was associated with increased and sustained amygdala and sad mood reactivity to the negative mood induction, even after controlling for potential confounds such as an influence of recent negative life events. This observation could reflect increased emotional reactivity, possibly as a result of adaptations to early stress in this group [49].

That said, ELS participants in our sample had low levels of ELS. Their greater mood changes might be in contrast to individuals with higher levels of ELS, who were not measured, but in whom more blunted affect has often observed [50]. And higher amygdala reactivity was associated with decreased effects of ELS on depressive symptoms. These data suggest that while ELS may increase amygdala and emotional reactivity, this outcome may reflect a more adaptive response than the alternative - having low preserved amygdala reactivity, in never-depressed people.

Preserved robust amygdala reactivity could reflect automatic reactivity or more of an effortful engagement process. During sad memory recall, the more the left amygdala activated, the more purported regulatory areas such as the bilateral DLPFC [3], motor cortex [51], and striatum [52] also activated in individuals with abuse history. Considering that patients with a major depressive disorder showed the reduced connectivity of the left amygdala with the cortical regions linked to top-down regulation [53], these data could suggest that preserved amygdala activity in healthy individuals reflects a willful or effortful engagement with emotional material, where a less regulated individual would have a more bluntedor dampened-affect presentation.

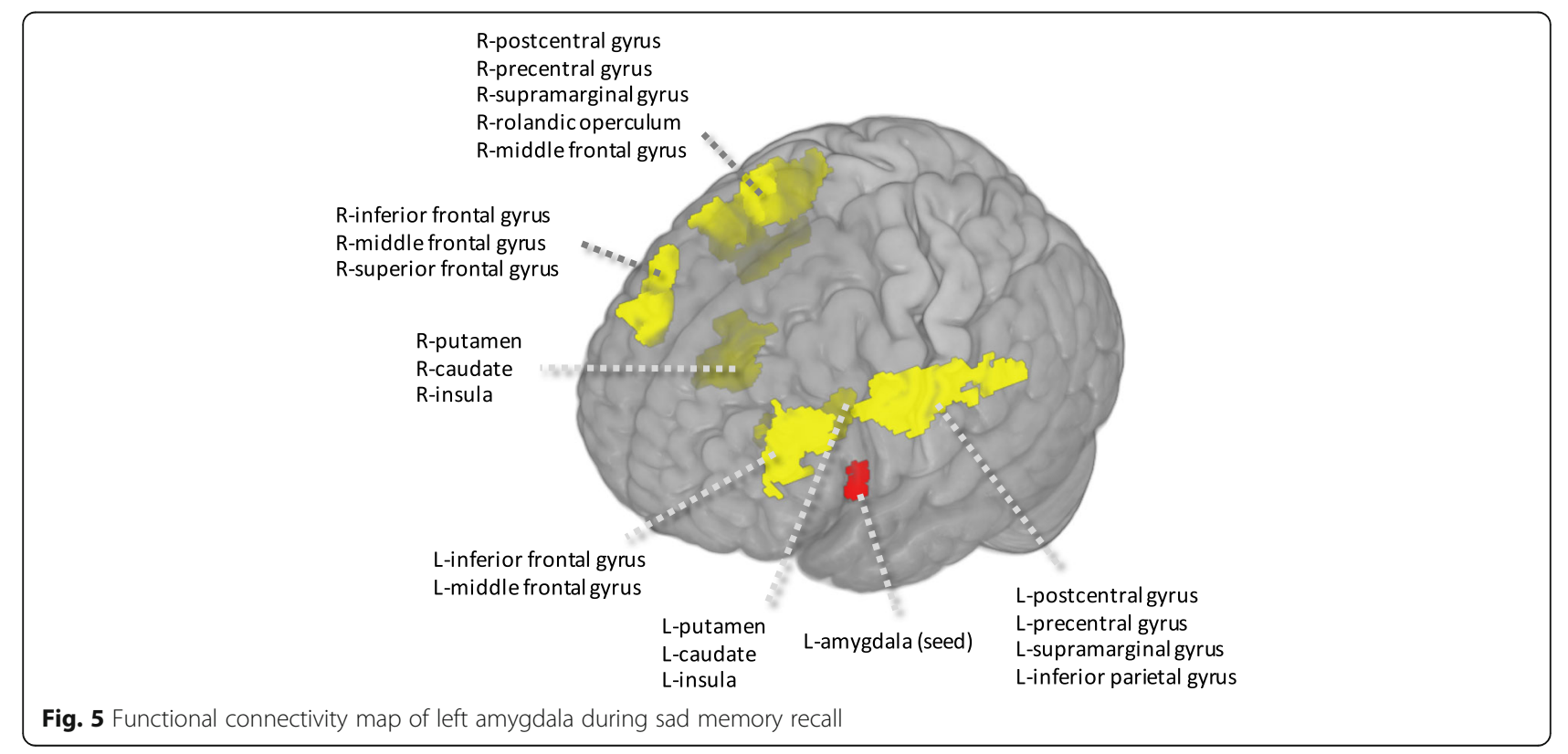


Thus we have speculated on a potential protective effect of preserved amygdala activation in resilient adults with ELS. These data could indicate that preserved reactivity in response to early life stress, may indicate increased ability to react to and process emotional information, which may be more adaptive than blunting strategies such as shutting down or avoiding. Increased amygdala activity was generally apparent for individuals with an ELS history. This pattern of increased reactivity in response to stress is well documented in both animal and human literature [54] and has been observed to precede more detrimental apathetic reactions that occur once an individual has given up hope, e.g., as in learned helplessness/hopelessness [55, 56]. While increased amygdala activity following early stress could be beneficial for people who are resilient to depression, as compared to a more blunted style, the same pattern could be problematic for depressed people, e.g., as it is associated with rumination in depression [57]. Therefore, accounting for a history of ELS and amygdala reactivity may be useful in helping to understand and promote resilience. In individuals with a history of ELS, prior to development of disorder it may be useful to work to increase reactivity to emotional information to increase resilience. For example, techniques such as compassion meditation, which is designed to enhance compassionate feelings, can increase amygdala response to negative images [58]. In this study, increased amygdala activation was correlated with decreased depression scores in the compassion meditation group composed of healthy adults, which suggests that in some cases, increased amygdala reactivity may also be beneficial for oneself.

This study had a number of limitations. We used a self-report measure to evaluate ELS. Given observed relationships between current mood and memory recall bias [59], future studies may benefit from a prospective analysis of the relationship between ELS and subsequent changes in amygdala activity, or at least, interview based measures of childhood maltreatment or documented cases of ELS. The sample size of this study was relatively small and there were moderate, if non-significant differences between groups on multiple demographic and clinical variables. Future studies could benefit from larger and more diverse sample sizes to confirm relationships between ELS and potentially protective effects of amygdala activity, above and beyond other clinical and demographic features. Moreover, all our participants were healthy subjects with no history of depression to exclude the confounding factor of psychiatric history on ELS. To substantiate the protective role of the amygdala, future studies should include participants with a similar ELS history, but also with a history of depression as a control group. Finally, scores for ELS in this study were in a range described by our measure's authors as the mild to moderate range. Thus, we cannot extend our inferences to higher levels of ELS.

\section{Conclusions}

In conclusion, our results suggest that 1) ELS leads to increased amygdala reactivity in healthy people in adulthood, and 2) this reactivity could be a protective factor for depression and recent negative stressful events. If these findings were replicated with an appropriate control group, interventions to increase amygdala activity in individuals with a history of ELS may be useful for prevention of depression.

\section{Abbreviations}

CATS: Child abuse and trauma scale; ELS: Early life stress; LES: Life event stress scale; PHQ-9: Patient health questionnaire-9; VAS: Visual analog scale

\section{Acknowledgments}

We thank the MRI staff at the Hiroshima City General Rehabilitation Center for use of facilities and technical support. Special thanks are due to Y. Ueno for his contribution to the project. We thank T. Yamamura, Y. Kaichi, M. Nishimoto, S. Nakamura, T. Nakatsukasa, Y. Miyamoto, and E. Watari for help with data acquisition as well as all subjects who participated in the study.

\section{Funding}

This work was supported by a Grant-in-Aid for 'Integrated research on neuropsychiatric disorders(15dm0107010h0005)' and 'Integrated Research on Depression, Dementia and Development Disorders(16dm0107093h0001)' carried out under the Strategic Research Program for Brain Sciences by AMED, and Grantin-aid for JSPS Research Fellows (25-2106) and JSPS KAKENHI Grant Number JP16H07011 to Tetsuya Yamamoto and MH096334 to Greg Siegle. None of the funding sources had any role in study design; in the collection, analysis and interpretation of data; in the writing of the report; and in the decision to submit the paper for publication.

\section{Availability of data and materials}

All data can be obtained from researchers by contacting the corresponding author.

\section{Authors' contributions}

Conceived and designed the experiments: TY ST MT S. Yoshimura TN YO S. Yamawaki. Performed the experiments: TY ST MT YT. Analyzed the data: TY ST GS MT. Wrote the paper, contributed to and have approved the final manuscript: TY ST GS MT YT S. Yoshimura GO TM TN HM YK T. Murakami YO S. Yamawaki.

\section{Competing interests}

The authors declare that they have no competing interests.

\section{Consent for publication}

Not applicable.

\section{Ethics approval and consent to participate}

The study was approved by the Research Ethics Committee of Hiroshima University. After complete description of the study to the participants, written informed consent was obtained.

\section{Author details}

${ }^{1}$ Department of Psychiatry, University of Pittsburgh School of Medicine, 121 Meyran Avenue, Loeffler Building, 15260-5003 Pittsburgh, PA, USA. ${ }^{2} J a p a n$ Society for the Promotion of Science, 8 Ichiban-cho, Chiyoda-ku, Tokyo 102-8472, Japan. ${ }^{3}$ Department of Psychiatry and Neurosciences, Institute of Biomedical and Health Sciences, Hiroshima University, 1-2-3 Kasumi, Minami-ku, 734-8551 Hiroshima, Japan. ${ }^{4}$ Western Psychiatric Institute and Clinic, 3811 O Hara St, 15213-2593 Pittsburgh, PA, USA. ${ }^{5}$ Faculty of Psychology, Otemon Gakuin University, 2-1-15 Nishiai, 567-8502 Ibaraki, Osaka, Japan. ${ }^{6}$ Department of Psychology, Graduate School of Education, Hiroshima University, 1-1-1 Kagamiyama, 739-8524 Higashi-Hiroshima, 
Hiroshima, Japan. ${ }^{7}$ Faculty of Health Sciences, Tsukuba International University, 6-20-1 Manabe, 300-0051 Tsuchiura, Ibaraki, Japan. ${ }^{8}$ Department of Radiology, Hiroshima City General Rehabilitation Center, 1-39-1 Tomo-minami, Asaminami-ku, 731-3168 Hiroshima, Japan. ${ }^{9}$ Kure Kyosai Hospital, 2-3-28 Nishi-chuo, 737-8505 Kure, Hiroshima, Japan. ${ }^{10}$ Present address. Graduate School of Integrated Arts and Sciences, Tokushima University 1-1, Minamijosanjima-cho, 770-8502 Tokushima, Japan.

Received: 15 September 2016 Accepted: 7 January 2017

Published online: 18 January 2017

\section{References}

1. Drevets WC. Prefrontal cortical-amygdalar metabolism in major depression. Ann N Y Acad Sci. 1999;877:614-37.

2. Drevets WC, Videen TO, Price $J$, Preskorn SH, Carmichael ST, Raichle ME. A functional anatomical study of unipolar depression. J Neurosci. 1992;12: 3628-41.

3. Siegle GJ, Thompson W, Carter CS, Steinhauer SR, Thase ME. Increased amygdala and decreased dorsolateral prefrontal BOLD responses in unipolar depression: related and independent features. Biol Psychiatry. 2007;61:198209.

4. Siegle GJ, Steinhauer SR, Thase ME, Stenger VA, Carter CS. Can't shake that feeling: event-related fMRI assessment of sustained amygdala activity in response to emotional information in depressed individuals. Biol Psychiatry. 2002;51:693-707

5. Monk CS, Klein RG, Telzer EH, Schroth EA, Mannuzza S, Moulton JL, et al. Amygdala and nucleus accumbens activation to emotional facial expressions in children and adolescents at risk for major depression. Am J Psychiatry. 2008;165:90-8

6. Ramel W, Goldin PR, Eyler LT, Brown GG, Gotlib IH, McQuaid JR. Amygdala Reactivity and Mood-Congruent Memory in Individuals at Risk for Depressive Relapse. Biol Psychiatry. 2007;61:231-9.

7. Pechtel P, Pizzagalli DA. Effects of early life stress on cognitive and affective function: An integrated review of human literature. Psychopharmacology (Berl). 2011;214:55-70.

8. Groenewold NA, Roest AM, Renken RJ, Opmeer EM, Veltman DJ, van der Wee NJ, et al. Cognitive vulnerability and implicit emotional processing: imbalance in frontolimbic brain areas? Cogn Affect Behav Neurosci. 2014;15: 69-79.

9. Blackford JU, Avery SN, Cowan RL, Shelton RC, Zald DH. Sustained amygdala response to both novel and newly familiar faces characterizes inhibited temperament. Soc Cogn Affect Neurosci. 2011;6:621-9.

10. Grant MM, Cannistraci C, Hollon SD, Gore J, Shelton R. Childhood trauma history differentiates amygdala response to sad faces within MDD. J Psychiatr Res. 2011;45:886-95.

11. Taylor SE, Eisenberger NI, Saxbe D, Lehman BJ, Lieberman MD. Neural Responses to Emotional Stimuli Are Associated with Childhood Family Stress. Biol Psychiatry. 2006;60:296-301.

12. Lupien SJ, Parent S, Evans AC, Tremblay RE, Zelazo PD, Corbo V, et al. Larger amygdala but no change in hippocampal volume in 10-year-old children exposed to maternal depressive symptomatology since birth. Proc Natl Acad Sci U S A. 2011;108:14324-9.

13. Newcomb MD, Munoz DT, Carmona JV. Child sexual abuse consequences in community samples of Latino and European American adolescents. Child Abuse Negl. 2009;33:533-44.

14. Murgatroyd C, Spengler D. Epigenetics of early child development. Front Psych. 2011;2:16.

15. Teicher MH, Andersen SL, Polcari A, Anderson CM, Navalta CP, Kim DM. The neurobiological consequences of early stress and childhood maltreatment. Neurosci Biobehav Rev. 2003;27:33-44.

16. Heim C, Nemeroff CB. The role of childhood trauma in the neurobiology of mood and anxiety disorders: preclinical and clinical studies. [Internet] Biol Psychiatry. 2001.

17. Heim C, Newport DJ, Heit S, Graham YP, Wilcox M, Bonsall R, et al. Pituitaryadrenal and autonomic responses to stress in women after sexual and physical abuse in childhood. J Am Med Assoc. 2000;284:592-7.

18. Pine DS, Cohen P, Brook JS. Emotional reactivity and risk for psychopathology among adolescents. CNS Spectr. 2001;6:27-35.

19. Kraus A, Esposito F, Seifritz E, Di Salle F, Ruf M, Valerius G, et al. Amygdala Deactivation as a Neural Correlate of Pain Processing in Patients with
Borderline Personality Disorder and Co-Occurrent Posttraumatic Stress Disorder. Biol Psychiatry. 2009;65:819-22.

20. Bylsma LM, Morris BH, Rottenberg J. A meta-analysis of emotional reactivity in major depressive disorder. Clin Psychol Rev. 2008;28:676-91.

21. McTeague LM, Lang PJ. The anxiety spectrum and the reflex physiology of defense: From circumscribed fear to broad distress. Depress Anxiety. 2012;29:264-81.

22. Boyce WT, Ellis BJ. Biological sensitivity to context: I. An evolutionarydevelopmental theory of the origins and functions of stress reactivity. Dev. Psychopathol. 2005;17:271-301.

23. Cuijpers P, Smit F. Subthreshold depression as a risk indicator for major depressive disorder: A systematic review of prospective studies. Acta Psychiatr Scand. 2004;109:325-31.

24. Otsubo T, Tanaka K, Koda R, Shinoda J, Sano N, Tanaka S, et al. Reliability and validity of Japanese version of the Mini-International Neuropsychiatric Interview. Psychiatry Clin Neurosci. 2005;59:517-26.

25. Sheehan DV, Lecrubier $Y$, Sheehan $\mathrm{KH}$, Amorim $\mathrm{P}$, Janavs J, Weiller $\mathrm{E}$, et al. The Mini-International Neuropsychiatric Interview (M.I.N.I.): The development and validation of a structured diagnostic psychiatric interview for DSM-IV and ICD-10. J Clin Psychiatry. 1998;59:22-33.

26. Oldfield RC. The assessment and analysis of handedness: the Edinburgh inventory. Neuropsychologia. 1971;9:97-113.

27. Beck AT, Steer RA, Brown GK. Manual for the Beck depression inventory-II. San Antonio, TX Psychol. Corp. 1996; 1-82.

28. Kojima M, Furukawa TA, Takahashi H, Kawai M, Nagaya T, Tokudome S. Cross-cultural validation of the Beck Depression Inventory-II in Japan. Psychiatry Res. 2002;110:291-9.

29. Matsuoka K, Uno M, Kasai K, Koyama K, Kim Y. Estimation of premorbid IQ in individuals with Alzheimer's disease using Japanese ideographic script (Kanji) compound words: Japanese version of National Adult Reading Test. Psychiatry Clin Neurosci. 2006;60:332-9.

30. Nelson HE, O'Connell A. Dementia: the estimation of premorbid intelligence levels using the New Adult Reading Test. Cortex. 1978;14:234-44.

31. Sanders B, Becker-Lausen E. The measurement of psychological maltreatment: Early data on the child abuse and trauma scale. Child Abuse Negl. 1995;19:315-23.

32. Tanabe H, Ozawa S, Goto K. Psychometric properties of the Japanese version of the Child Abuse and Trauma Scale (CATS). In: 9th Annu. Meet. Japanese Soc. Trauma. Stress Stud. (in Japanese). 2010.

33. Kent A, Waller $\mathrm{G}$. The impact of childhood emotional abuse: An extension of the child abuse and trauma scale. Child Abuse Negl. 1998;22:393-9.

34. Nakai $Y$, Toda $H$, Inoue T, Toyomaki A, Tanaka T, Nakagawa S, et al. A study of validation and reliability of the Japanese version of the Life Experiences Survey. 108th Annu Meet Japanese Soc Psychiatry Neurol. Sapporo (in Japanese). 2012

35. Sarason IG, Johnson JH, Siegel JM. Assessing the impact of life changes: development of the Life Experiences Survey. J Consult Clin Psychol. 1978;46:932-46

36. Kroenke K, Spitzer RL, Williams JBW. The PHQ-9: Validity of a brief depression severity measure. J Gen Intern Med. 2001;16:606-13.

37. Muramatsu K, Miyaoka H, Kamijima K, Muramatsu Y, Yoshida M, Otsubo T, et al. The patient health questionnaire, Japanese version: validity according to the mini-international neuropsychiatric interview-plus. Psychol Rep. 2007;101:952-60.

38. Segal ZV, Gemar M, Williams S. Differential cognitive response to a mood challenge following successful cognitive therapy or pharmacotherapy for unipolar depression. J Abnorm Psychol. 1999;108:3-10.

39. Gemar MC, Kapur S, Segal ZV, Brown GM, Houle S. Effects of self-generated sad mood on regional cerebral activity: A PET study in normal subjects. Depression. 1996:4:81-8

40. Eich E, Ng JTW, Macaulay D, Percy AD, Grebneva I. Combining music with thought to change mood. In: Coan JA, Allen JJB, Coan JA, Allen JJB, editors. Handb. Emot. elicitation Assess. New York: Oxford University Press; 2007. p. 124-36.

41. Mitterschiffthaler MT, Fu CHY, Dalton JA, Andrew CM, Williams SCR. A functional MRI study of happy and sad affective states induced by classical music. Hum Brain Mapp. 2007;28:1150-62.

42. Eugène $F$, Lévesque J, Mensour B, Leroux J-M, Beaudoin $G$, Bourgouin $P$, et al. The impact of individual differences on the neural circuitry underlying sadness. Neuroimage. 2003;19:354-64.

43. Tzourio-Mazoyer N, Landeau B, Papathanassiou D, Crivello F, Etard O, Delcroix N, et al. Automated anatomical labeling of activations in SPM using 
a macroscopic anatomical parcellation of the MNI MRI single-subject brain. Neuroimage. 2002;15:273-89.

44. Maldjian JA, Laurienti PJ, Kraft RA, Burdette JH. An automated method for neuroanatomic and cytoarchitectonic atlas-based interrogation of fMRI data sets. Neuroimage. 2003;19:1233-9.

45. Brett M, Anton J-LL, Valabregue R, Poline J-B. Region of interest analysis using an SPM toolbox [abstract] Presented at the 8th International Conference on Functional Mapping of the Human Brain, June 2-6, 2002, Sendai, Japan. Neuroimage. 2002;16:abstract 497.

46. McLaren DG, Ries ML, Xu G, Johnson SC. A generalized form of contextdependent psychophysiological interactions (gPPI): A comparison to standard approaches. Neuroimage. 2012;61:1277-86.

47. Cox RW. AFNl: software for analysis and visualization of functional magnetic resonance neuroimages. Comput Biomed Res. 1996;29:162-73.

48. Dannlowski U, Stuhrmann A, Beutelmann V, Zwanzger P, Lenzen T, Grotegerd D, et al. Limbic scars: long-term consequences of childhood maltreatment revealed by functional and structural magnetic resonance imaging. Biol Psychiatry. 2012;71:286-93.

49. McCrory E, De Brito S, Viding E. The link between child abuse and psychopathology: a review of neurobiological and genetic research. J R Soc Med. 2012;105:151-6.

50. Daversa MT. Early environmental predictors of the affective and interpersonal constructs of psychopathy. Int J Offender Ther Comp Criminol. 2010;54:6-21.

51. Vrticka P, Simioni S, Fornari E, Schluep M, Vuilleumier P, Sander D. Neural substrates of social emotion regulation: A fMRI study on imitation and expressive suppression to dynamic facial signals. Front Psychol. 2013;4:95.

52. Voytek B, Knight RT. Prefrontal cortex and basal ganglia contributions to visual working memory. Proc Natl Acad Sci USA. 2010;107:18167-72.

53. Ramasubbu R, Konduru N, Cortese F, Bray S, Gaxiola-Valdez I, Goodyear B. Reduced intrinsic connectivity of amygdala in adults with major depressive disorder. Front Psych. 2014;5:1-11.

54. Davis M, Whalen PJ. The amygdala: vigilance and emotion. Mol Psychiatry. 2001;6:13-34.

55. Abramson LY, Seligman ME, Teasdale JD. Learned helplessness in humans: critique and reformulation. J Abnorm Psychol. 1978;87:49-74.

56. Forgeard MJC, Haigh EAP, Beck AT, Davidson RJ, Henn FA, Maier SF, et al. Beyond depression: Toward a process-based approach to research, diagnosis, and treatment. Clin Psychol Sci Pract. 2011:18:275-99.

57. Mandell D, Siegle GJ, Shutt L, Feldmiller J, Thase ME. Neural substrates of trait ruminations in depression. J Abnorm Psychol. 2014;123:35-48.

58. Desbordes G, Negi LT, Pace TWW, Wallace BA, Raison CL, Schwartz EL. Effects of mindful-attention and compassion meditation training on amygdala response to emotional stimuli in an ordinary, non-meditative state. Front Hum Neurosci. 2012;6:292.

59 Matt GE, Vazquez C, Campbell WK. Mood-congruent recall of affectively toned stimuli: A meta-analytic review. Clin Psychol Rev. 1992;12:227-55.

\section{Submit your next manuscript to BioMed Central and we will help you at every step:}

- We accept pre-submission inquiries

- Our selector tool helps you to find the most relevant journal

- We provide round the clock customer support

- Convenient online submission

- Thorough peer review

- Inclusion in PubMed and all major indexing services

- Maximum visibility for your research

Submit your manuscript at www.biomedcentral.com/submit

CBiomed Central 